\section{APPLICATION OF GRAPHENE BASED COATING ON CORONARY ARTERY STENTS}

Fatemeh Jafarzadeh*, Daryl McManus, Irina Barbolina, Nadim Malik, Cinzia Casiraghi, Cathy Holt. University of Manchester

\subsection{6/heartinl-2017-311726.144}

Coronary artery disease is the leading cause of death worldwide. Stent implantation is the mainstay approach to revascularise stenosed coronary arteries. Bare metal stents were the first stents designed, but presented a restenosis risk of approximately $20 \%$ of patients due to restenosis. Subsequently, drug eluting stents were introduced, which, however, introduced late in stent thrombosis. We propose the use of a graphene based coating onto bare metal stents in an attempt to significantly reduce stent associated complications and promote vessel healing. Graphene is a single layer of carbon atoms arranged in a honeycomb lattice. The unique properties of graphene make it an ideal material to use as an implantable device coating: It has a high surface to volume ratio; it is impermeable and atomically smooth and has been shown to exhibit bio-compatible properties. Graphene based dispersions were prepared by liquid phase exfoliation in water. Investigations to coat bare metal stents were undertaken. Dip, spin and spray coating methods were explored. Raman spectroscopy was measured to identify and characterise the coated material. Raman spectroscopy demonstrated spray coating to result in the most uniform and thin graphene based coating. In addition, human endothelial cell adherence and proliferation on the graphene based coating was studied. Hoechst 33342 and phalloidin stains were used to image the cells under fluorescence microscopy. This revealed the adherence of human endothelial cells to be unaffected by the graphene based coating. In conclusion, spray coating created the most uniform and thin graphene based coating onto bare metal stents. Human coronary artery endothelial cell adherence occurred on the graphene coated stents. Future work is aimed at studying bio- and haemo- compatibility of graphene based coating and their performance in a porcine stent model.

\section{FREE FETAL HAEMOGLOBIN IMPACTS FETOPLACENTAL VASCULAR STRUCTURE AND FUNCTION: IMPLICATIONS FOR FETAL GROWTH RESTRICTION}

\begin{abstract}
${ }^{1}$ Adam Brook*, ${ }^{2}$ Rosie Sneyd, ${ }^{3}$ Rekha Gurung, ${ }^{4}$ Stefan Hansson, ${ }^{3}$ lan Crocker, ${ }^{3}$ Paul Brownbill. 'Maternal and Fetal Health Research Centre, Institute of Human Development, University of Manchester, and Central Manchester NHS Foundation Trust; ${ }^{2}$ Maternal and Fetal Health Research Centre, University of Manchester, and Central Manchester NHS Foundation Trust, ${ }^{3}$ Maternal and Fetal Health Research Centre and Central Manchester University Hospitals NHS Foundation Trust; ${ }^{4}$ Department of Obstetrics and Gynaecology, Institute of Clinical Sciences, Lund University
\end{abstract}

10.1136/heartjnl-2017-311726.145

Introduction In fetal growth restriction (FGR), early placentation defects result in placental insufficiency and vasoconstriction, which cumulate in organ failure at the severe end of the spectrum. In other placental pathologies, including preeclampsia, extracellular free fetal haemoglobin $(\mathrm{fHbF})$ is overproduced, where it inactivates the vasodilator nitric oxide (NO). We have previously shown that excess fetoplacental $\mathrm{fHbF}$ also occurs in FGR pregnancy, sequestering $\mathrm{NO}$ and evoking vasoconstriction. Here, we explore the further effects of $\mathrm{fHbF}$ on fetoplacental endothelium, considering altered angiogenesis, altered vasculoprotection, and an immunological response.

Methods We assessed the $\mathrm{fHbF}$ evoked synthesis of the proangiogenic and pro-inflammatory cell stress mediators DKK-4, NFkB and FABP-1, produced by human chorionic plate arterial endothelial cells (HPAECs) under conditions of unidirectional laminar flow. Secondly, we studied fHbF-evoked $\mathrm{NFA \tilde {A }} \square \hat{\mathrm{A}}^{\circ} \mathrm{B}$ nuclear translocation using fluorescent microscopy; and the downstream NFkB-actuated cytokine profile (IF1 $\alpha$, $\mathrm{TNF} \alpha$ ) measured in cell medium and lysate by ELISA. Observations were expanded to assessment of $\mathrm{fHbF}$ effects on branching and non-branching angiogenesis of HPAECs grown on Matrigel, and expressed as an average of tubule length multiplied by tubule number as a net measure of angiogenesis. As a correlate, endothelial morphology of $\mathrm{fHbF}$ and nonexposed static chorionic artery sections was qualitatively analysed by scanning electron microscopy.

Results A cell stress proteome assay demonstrated that $\mathrm{fHbF}$ increased synthesis of DKK-4 (>50\% increase from control), NFKB (>50\% increase) and FABP1 (>100\% increase). A proangiogenic effect was further corroborated by assessment of villous angiogenesis on Matrigel, where $0.3 \mathrm{mg} / \mathrm{ml} \mathrm{fHbF}$ was found to promote branching angiogenesis in HPAECs $(n=5$; $\mathrm{p}<0.01$ ). In a separate study of $\mathrm{NF \kappa B}$-mediated endothelial inflammation, $0.3 \mathrm{mg} / \mathrm{ml} \mathrm{fHbF}-$ evoked $\mathrm{NFkB}$ signal transduction demonstrable by nuclear translocation within 2.5 hours, whilst in controls no evidence of NFkB activation was observed ( $n=4$ paired lines). In the $\mathrm{fHbF}$ group this was paralleled by increased release of NFKB-actuated cytokines into flow-conditioned medium, IFN1 $\pm(n=7$; control vs. fHbFexposed at $0.2 \mathrm{mg} / \mathrm{ml} \mathrm{fHbF}$ median fold change 1.42 (range 1.09-2.2) vs. 1 (0); Wilcoxon $\mathrm{p}<0.05)$, and for $\mathrm{TNF} \alpha$ (control vs. fHbF-exposed at $0.2 \mathrm{mg} / \mathrm{ml} \mathrm{fHbF}$ median foldchange 1.54 (range 1.09-2.22) vs. 1 (0); Wilcoxon $\mathrm{p}<0.05$ ). Morphologically, $\mathrm{fHbF}$ evoked widespread blebbing of the luminal endothelium.

Conclusion $\mathrm{fHbF}$ causes aberrant pro-angiogenesis, promotes acute inflammation and loss of structural organisation in fetoplacental endothelium. Our findings are relevant to widening understanding the pathophysiology of FGR and exploring the link between FGR and stillbirth, where overproduction of extracellular fetal haemoglobin could represent a novel therapeutic target.

\section{PLATELET COX-1 KNOCKOUT MOUSE AS A MODEL OF THE EFFECTS OF ASPIRIN IN THE CARDIOVASCULAR SYSTEM}

${ }^{1}$ Marilena Crescente* ${ }^{*}{ }^{1}$ Paul C Armstrong, ${ }^{1}$ Melissa V Chan, ${ }^{2}$ Matthew L Edin, ${ }^{2}$ Fred B Lih, ${ }^{3}$ J Jiao, ${ }^{1} \mathrm{C}$ Gaston-Massuet, ${ }^{4} \mathrm{GS}$ Cottrell, ${ }^{5} \mathrm{NS}$ Kirkby, ${ }^{5} \mathrm{JA}$ Mitchell, ${ }^{2} \mathrm{DC}$ Zeldin, ${ }^{3} \mathrm{HR}$ Herschman, ${ }^{1}$ Timothy D Warner. ${ }^{1}$ Queen Mary University of London; ${ }^{2}$ NIH/NIEHS: ${ }^{3}$ UCLA; ${ }^{4}$ University of Reading; ${ }^{5}$ mperial College London

10.1136/heartjnl-2017-311726.146 
Introduction Aspirin acts as an anti-thrombotic drug by inhibiting platelet cyclooxygenase- 1 (COX-1) and blocking the production of thromboxane A2, but it also has off-platelet actions that may limit its effectiveness. Since mice have very marked differences in aspirin pharmacokinetics they cannot be used to explore the effects of low dose aspirin in humans. To address these questions, we developed platelet COX-1 knockout mice (platelet-COX-1 ${ }^{-/-}$) to recapitulate the effect of low-dose aspirin in humans.

Methods and Results. Cox $-1^{f l / f l}$ mice were paired with Pf4-Cre mice to delete COX-1 in the MK lineage. The absence of COX-1 in platelets was confirmed by Western blot analysis, confocal immunofluorescence microscopy and by analysing the distribution of the COX-1 signal and mass with an highthroughput confocal imaging system. The production of thromboxane $\mathrm{B} 2\left(\mathrm{TxB}_{2}\right)$ induced by collagen, $\mathrm{A} 23187$ and the PAR-4 activating peptide in platelet rich plasma and whole blood was significantly decreased in platelet-COX-1 ${ }^{-/}$mice. This effect was not further reduced by the addition of aspirin in vitro. Further eicosanomic analysis was performed using LC-MS/MS of samples from untreated and A23187-stimulated whole blood. 11- and 15-hydroxyeicosatetraenoic acid (HETE), alongside $\mathrm{TxB}_{2}$ formation were inhibited after platelet COX-1 deletion. Production of 12-HETE, coming from 12-lipoxygenase (LOX), and 5-HETE coming from leukocyte 5 -LOX, was unchanged in platelet-COX-1 $1^{-/-}$mice. To better dissect the effects of aspirin in vivo, we used a thrombosis model caused by ferric chloride injury in the mouse carotid artery. Platelet $\mathrm{COX}-1^{-/-}$mice, that recapitulate the effects of low-dose aspirin, formed unstable thrombi that were embolizing before producing a stable vessel occlusion. Interestingly, when a high dose of aspirin $(10 \mathrm{mg} / \mathrm{kg})$ was injected into the mice, the thrombus was stable and formed earlier than in the vehicle-treated mice.

Conclusions We have produced the first platelet-COX-1 ${ }^{-/}$ mouse and characterised its phenotype and eicosanomic profile. This model is allowing us to mechanistically dissect the platelet and non-platelet effects of aspirin and so better model the effects of clinically important anti-thrombotic therapy.

\section{PAEDIATRIC CARDIAC ARREST: IS THERE ANY MYOCARDIAL DAMAGE IN PAEDIATRIC PATIENTS WHEN ADULT DEFIBRILLATION ENERGIES ARE ADMINISTERED?}

Laura Davis*, ${ }^{1}$ Rebecca Funston, Paul Crawford, ${ }^{1}$ Olibhar McAlister, ${ }^{1}$ Ben McCartney, ${ }^{1}$ Hannah Torney, ${ }^{2}$ Alistair Courtney, ${ }^{3}$ Rachael Gregson, ${ }^{4}$ David McEneaney, ${ }^{3}$ Eddie Clutton, ${ }^{5}$ Jennifer Adgey. ${ }^{1}$ Physio-Control; ${ }^{2}$ Ulster University; ${ }^{3}$ University of Edinburgh; ${ }^{4}$ Craigavon Area Hospital; ${ }^{5}$ Royal Victoria Hospital

\subsection{6/heartjnl-2017-311726.147}

Purpose When attempting defibrillation in an out-of-hospital paediatric patient, the use of a reduced energy is currently recommended in the resuscitation guidelines due to concerns surrounding potential myocardial damage. The purpose of this pilot study was to investigate the 1 st shock success of two different automated external defibrillator (AED) energy protocols and observe the resulting levels of myocardial damage in a paediatric model of cardiac arrest.

Method A total of six piglets $(10-25 \mathrm{~kg})$ were anaesthetised, instrumented and ventricular fibrillation (VF) was electrically induced. After approximately $15 \mathrm{~s}$ of untreated VF, a defibrillation shock was delivered using the selected device in line with Protocol A (adult energy doses of 150J) or Protocol B (paediatric energy doses of 75J). Following successful defibrillation, a $3 \mathrm{~min}$ recovery period was allowed for the heart to recover before $\mathrm{VF}$ was again induced with a maximum of 20 shocks administered. Blood samples were collected at predefined time-points whilst the animal was under anaesthesia and analysed for cardiac troponin I (cTnI). After the defibrillation phase of the protocol, the animals remained anaesthetised and vital signs monitored for a period of 6 hours with blood samples collected hourly for further analysis.

Results A total of 120 shocks were delivered across both protocols with a 100\% shockable rhythm detection and 100\% first shock success observed in both Protocol A and B. Prior to the induction of VF, Protocol A and B presented with similar initial mean $( \pm S D)$ levels of cTnI; $0.04( \pm 0.03) \mathrm{ng} / \mathrm{ml}$ $(\mathrm{n}=3)$ and $0.03( \pm 0.02) \mathrm{ng} / \mathrm{ml}(\mathrm{n}=3)$, respectively. Comparable results of cTnI were also observed upon completion of the defibrillation phase (Shock 20); Protocol A - $0.12( \pm 0.05)$ $\mathrm{ng} / \mathrm{ml}$ and Protocol B $-0.14( \pm 0.07) \mathrm{ng} / \mathrm{ml}$. An increase in mean cTnI was detected into the rest period, peaking by 5 hours for both energy protocols. At the 6 hour endpoint of this study, the mean value of cTnI in the blood was reducing for both energy protocols with no statistically significant difference observed between the high and low defibrillation energy groups.

Conclusion There was no statistical evidence to suggest that treating the paediatric animals with a higher adult shock energy caused more myocardial damage than a paediatric shock energy. Further investigations are warranted to determine the long term impact on myocardial tissues in a paediatric patient.

\section{RAISED INTRACELLULAR CA ${ }^{2+}$ CONCENTRATION BY PERSISTENT NA ${ }^{+}$CHANNELS ACTIVATION DISRUPTS CELLULAR CIRCADIAN RHYTHMICITY OF BMAL1 CLOCK GENE IN CULTURED ATRIAL MYOCYTES}

Andria Siakalli* ${ }^{*}$ Daan van der Veen, Rita Jabr. University of Surrey

10.1136/heartjnl-2017-311726.148

One of the main contributor to atrial arrhythmias (AA) is the activation of the persistent $\mathrm{Na}$ channels $\left(\mathrm{Na}_{\mathrm{P}}\right)$ leading to raised intracellular $\mathrm{Ca}^{2+}$ concentration $\left(\left[\mathrm{Ca}^{2+}\right]_{\mathrm{i}}\right)$. Incidences of AA exhibit diurnal patterns suggestive of possible correlation between circadian rhythms and atrial electrophysiology. Circadian rhythms are mediated by cellular circadian clocks, made up of transcriptional-translational feedback loops consisting of several genes, in particular Bmal1. So far, the correlation between raised $\left[\mathrm{Ca}^{2+}\right]_{i}$ induced by activated $\mathrm{Na}_{\mathrm{P}}$ and Bmal1 rhythmicity is unknown. The aim of this study is to assess the effects of 1) $\mathrm{Na}_{\mathrm{P}}$ opener, ATXII, Anemonia Sulcata toxin II and 2)raised $\left[\mathrm{Ca}^{2+}\right]_{i}$ induced by $\mathrm{Na}_{\mathrm{P}}$ on Bmal1 gene circadian rhythmicity in cultured mouse atrial (HL-1-6) cells and mouse embryonic fibroblasts (MEFs, positive control).

HL-1-6 were grown in Claycomb medium with 10\% FBS, $0.1 \mathrm{mM}$ norepinephrine and $2 \mathrm{mM}$ l-glutamine, whereas, MEFs were maintained in DMEM with 10\% FBS. The cells were transduced with Bmal1::luciferase (BMAL1::LUC) probe to determine bioluminescence rhythmicity through long-term bioluminescence recording over several circadian cycles (45 days). Cells were serum shocked (2 hours) using 50\% FBS to synchronise all cellular clocks. Cells were then incubated in 